\title{
Network representation with Clustering Tree Features
}

\author{
Konstantinos Pliakos • Celine Vens
}

Received: date / Accepted: date

\begin{abstract}
Representing and inferring interaction networks is a challenging and long-standing problem. Modern technological advances have led to a great increase in both volume and complexity of generated network data. The size of networks such as drug protein interaction networks or gene regulatory networks is constantly growing and multiple sources of information are exploited to extract features describing the nodes in such networks. Modern information systems need therefore methods that are able to mine these networks and exploit the available features. Here, a novel data mining framework for network representation and mining is proposed. It is based on decision tree learning and ensembles of trees. The proposed scheme introduces an efficient network data representation, capable of addressing different data types, tackling as well data volume and complexity. The learning process follows the inductive setup and it can be performed in both a supervised or unsupervised manner. Experiments were conducted on six biomedical network datasets. The experimental evaluation demonstrates the merits of the proposed approach, confirming its efficiency.
\end{abstract}

Keywords tree-ensembles, extremely randomized trees, interaction data representation, biomedical network mining, graph embedding

Konstantinos Pliakos

KU Leuven, Campus KULAK, Department of Public Health and Primary Care, Etienne Sabbelaan 53, Kortrijk 8500, Belgium

E-mail: konstantinos.pliakos@kuleuven.be

Celine Vens

KU Leuven, Campus KULAK, Department of Public Health and Primary Care, Etienne Sabbelaan 53, Kortrijk 8500, Belgium

E-mail: celine.vens@kuleuven.be 


\section{Introduction}

Nowadays, there is generally a great increase in data generation and use. These huge volumes of data are generated in terms of both cardinality (i.e., the number of samples) and dimensionality (i.e., the number of features that describe each sample). They are often characterized by complex structures, combining information from multiple sources. One example that is often encountered is interaction data. Instead of one set of objects described by a set of features, interaction data are characterized by two sets of objects, each described by its own set of features. Interaction data is omni-present: in social network analysis, recommender systems, ecology (habitat modeling), bioinformatics (gene expression analysis, drug response analysis, predicting drug-target reactions), technology-enhanced education, etc.

Relations between entities that interact with each other such as drugpatient interactions in medicine are often represented by networks (here, equally referred to as graphs). There are two types of networks, homogeneous and heterogeneous. Homogeneous networks model samples of the same type (e.g., protein-protein networks) while heterogeneous model samples of different type (e.g., drug-protein networks). Heterogeneous networks are also referred to as bi-partite graphs. The two interaction sets (e.g., drugs or proteins in drugprotein interaction networks) are described by their own features. The actual interactions between the instances of these two sets are represented as a matrix containing binary or numeric items $[1,2]$. In Fig. 1, an example of an interaction network is displayed. The values of interest are usually the interactions between instances. More precisely, in interaction prediction the goal is to predict if a possible connection between two instances exists. Despite the continuous rising in the amount of available data, usually we have only a very partial knowledge of these networks [3]. Thus, network representation methods that can improve the performance of machine learning and data mining techniques applied to these networks are needed.

Motivated by our previous work [4], here we propose an efficient data representation framework for network mining. The proposed approach can be applied to both homogeneous and heterogeneous interaction networks. It is based on ensembles of decision trees, inheriting the advantages of tree ensemble learning. Decision tree induction algorithms $[5,6]$ are among the most popular data mining algorithms. They have been applied extensively in many fields such as systems biology [7] or social media analysis [8]. A crucial advantage of these methods is the interpretability of the models they produce, making them transparent and understandable to human experts, also leveraging knowledge discovery. Other advantages include their scalability from a computational point of view and their fair predictive accuracy. Extending them to ensemble methods $[9,10]$ improves their predictive performance and provides state-of-the-art results.

The proposed approach employs Extremely Randomized Trees [11], hereafter denoted as ERT. In particular, every node of each decision tree of the ensemble was treated as a cluster, containing all the samples that fall into 
that tree node. Next, binary feature vectors are generated, where each component represents the presence or absence of a sample in a cluster (node). The new features are generated in an inductive manner (i.e., the test samples are not needed during learning). This way, the proposed approach has a substantial advantage over other feature construction approaches that follow the transductive setup.

We extend the unsupervised approach presented in [4] along several directions. In particular:

- We investigate the employment of a random sampling strategy on the output space.

- We extend our method by applying a filtering scheme on the nodes (clusters) of each tree.

- We test the employment of several dimensionality reduction methods $[13$, $14]$.

- The proposed data representation approach is extended towards interaction data in both an unsupervised and supervised way.

First, we investigate the employment of a random sampling strategy on the output space. While the random selection of the features during the training procedure is an essential characteristic of the ensemble-based algorithms (e.g., Random Forests, Extra Trees, etc.) the random sampling of the output space has not drawn the same level of attention. In [12], the idea of random projections applied to the output space was investigated in the context of multilabel classification. The experimental evaluation led to promising results as the authors managed to reduce the computational burden without harassing the accuracy of their models. An interesting question is thereby whether the same phenomenon holds in the unsupervised learning setup. A positive answer to this question could drastically increase the applicability of tree-based clustering methods on high dimensional data.

Furthermore, one disadvantage of the previous method presented in [4] was the large size of the induced feature set. Therefore, we extend our method by applying a filtering scheme on the nodes (clusters) of each tree based on the number of samples they contain. This way we create a smaller and therefore computationally more efficient feature set. In addition, we test the employment of several dimensionality reduction techniques [13,14], addressing the problem of the ever-increasing data volume. The efficiency in detecting an underlying manifold over complex data is also tested. By reducing the size of the feature vectors we achieve noise removal while maintaining relations between the samples (nodes of the network). We succeed in generating an informative, compact and computationally efficient feature representation.

Moreover, the proposed data representation approach is extended towards interaction data in both an unsupervised and supervised way. The differences between the two settings in network mining are highlighted. As it was discussed in the beginning of this section, data nowadays follow more complex structures and new methods to handle them are needed. When it comes to networks, the 


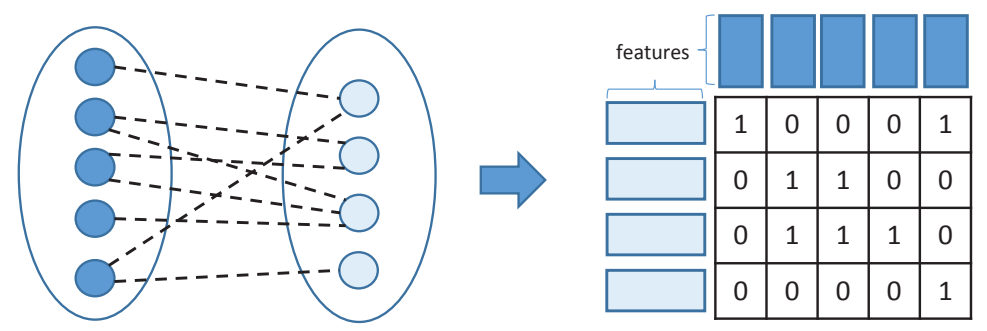

Fig. 1 Illustration of an (bi-partite) interaction network.

application of supervised learning methods is not trivial as different learningtesting settings emerge.

The outline of the paper is as follows. In section 2, related studies are discussed. In Section 3, the proposed approach is described in detail. The experimental evaluation is presented in Section 4. Conclusions are drawn and topics of future research are discussed in Section 5 .

\section{Related Work}

In the past, the performance of machine learning algorithms was improved by leveraging the mining of complex patterns through mapping the data to different feature spaces [15]. Most of the developed methods were based on kernel learning $[16,17]$, mainly due to the very good performance of Support Vector Machines (SVMs) [18]. However, these methods are often characterized by high computational costs and limited flexibility as one should compute and handle the whole Gram matrix. Many of these kernel-based methods have also been developed in a transductive setup where test instances are available during the training phase [16].

There are several studies where new features are constructed inductively using clustering techniques or decision tree learning. Most of the recently developed feature construction methods were developed for supervised learning tasks. In [19], a feature induction method based on random forests [10] was proposed. It was based on a metric transformation that mapped the identity of the tests performed in each node of a decision tree to a feature indicator. Feature vectors were generated by concatenating all the features corresponding to each tree in the forest and they were further encoded using hashing. A similar transformation of the data, using a set of random clustering forests was proposed in $[20,21]$ for visual codebook construction. In particular, the features were generated by randomized trees. The data encoding was based only on the indices of the leaves where a data sample ends up. The approach leads to a high dimensional, sparse binary coding. In [22], a label-specific feature scheme for multi-label classification was proposed. For each label, a distinct feature set was constructed by clustering the label's positive and negative instances (separately), and then calculating the distances of each instance to the obtained 
cluster centroids. This way, the predictive performance of a classifier trained for that specific label was increased. In [4], an unsupervised feature induction technique based on Extremely Randomized Trees [11] was presented. Target informed features based on Extremely randomized Trees were recently applied to boost variance between data instances in biomedicine [23]. The focus of that work was on multi-target prediction.

When it comes to network data, studies usually focus on network reconstruction. Both supervised and unsupervised machine learning methods have been used to complete a partially known network [24-26]. It was shown [27, 28] that particularly efficient are methods that combine the predictions of several algorithms in a consensus framework. Aiming to address the challenging problem of gene regulatory network reconstruction, a community approach containing over 30 methods was developed in [27]. More specifically, consensus networks were built over 30 network inference methods that were employed to predict regulatory interactions between transcription factors and target genes. The aggregate of all these interactions composed the general reconstruction of the network. Following a similar logic, an algorithm called TopkNet was introduced integrating $\mathrm{k}$ high-performance network inference algorithms in [28]. TopkNet was applied in combination with a quantitative measure of similarity among datasets. A semi-supervised method that learns to integrate the predictions from several inference methods was proposed in [29]. The method was based on ensemble learning. The interactions predicted by several network inference algorithms were integrated in a multi-view learning setup. Based also on this strategy a semi-supervised ensemble learning classifier learning to integrate miRNA-gene target interactions from multiple prediction models was combined with bi-clustering to build the core of a web-based database system called ComiRNet (Co-clustered miRNA Regulatory Networks) [30]. ComiRNet was developed to alleviate the discovery of miRNA functions by providing biomedical scientists with a repository of tools aiming at predicting interactions.

Besides the consensus based approaches, top results were also obtained by using methods that use a single algorithm. In [31], the authors presented GENIE3, a method that addresses the reconstruction of regulatory networks as the aggregation of multiple regression problems. GENIE3 predicts the expression pattern of each gene of the network by training a tree ensemble learner (ERT) on the patterns of the remaining genes. Extremely randomized trees were also used in [3]. The authors in [3] addressed the problem of network inference as a supervised learning task. They also performed a comparison study between 3 different learning settings, discussing their advantages and weaknesses.

Different from the approaches presented above, the method proposed in this paper does not focus on network reconstruction but on network representation. We propose a machine learning approach that generates a better network representation which can work as a solid base for subsequent network reconstruction or other network mining tasks. 


\section{Method}

Here, the proposed method is presented. First, theoretical background information is provided. Next, the basic feature induction method is described and the proposed network mining model is presented.

\subsection{Learning using Extremely Randomized Trees}

Decision trees are typically constructed with a top-down induction method. Starting from the root node that is associated with the complete training set, the nodes are recursively split by applying a test to one of the features. In order to find the best split, all features and their corresponding split points are considered and a split quality criterion is evaluated. In supervised learning tasks, this criterion is often information gain (classification), or variance reduction (regression). When the data contained in a node is pure w.r.t. the target, or when some other stopping criterion holds, the node becomes a leaf node and a prediction is assigned to it. This prediction is the majority class assigned to the training instances in the leaf for classification, or the average of their target values for regression. The prediction for test instances is obtained by sorting them through the tree into a leaf node.

In this work, the decision tree learners employed are set in the Predictive Clustering Tree (PCT) [6] framework, adopting the hierarchical clustering view of decision trees. PCTs are constructed by maximally reducing intra-cluster variance at each split. By computing the variance over the feature set, rather than the target, PCTs can be applied to (unsupervised) clustering tasks.

Since decision trees often have a large variance, their predictive performance can be improved by having several trees returning an aggregated prediction. Such a collection of decision trees is called an ensemble, and several instances of ensembles exist. In this work, we consider the ensemble method of Extremely Randomized Trees (ERT) [11,32]. The ERT algorithm builds an ensemble of unpruned decision trees following the traditional top-down procedure. In an ERT ensemble, each tree is constructed by considering only a random set of split candidates at each node. More precisely, a random subset of features is picked, and for each feature, a random split point is picked. From these candidates, the candidate yielding the best value for the split criterion is chosen. The growing of each tree is stopped when the tree is fully grown (i.e., one sample in each leaf) or a criterion has been reached (e.g., maximum depth, minimum number of samples to split, etc.).

The rationale behind the ERT algorithm is that the explicit randomization of the splitting threshold and attribute in combination with ensemble averaging reduces bias-variance more strongly than the randomization performed by other methods. ERT was shown to have a better predictive performance than the more popular Random Forests [11] and it is also computationally less expensive due to the simplicity of the node splitting procedure. 
3.2 Feature induction with extremely randomized trees

A new feature set is generated by applying ERT on the initial feature set, as follows. The nodes of each tree in the ERT setting, $\mathbf{Q}=\left\{q_{1}, q_{2}, \cdots, q_{|Q|}\right\}$ are treated as clusters containing all the samples that fall into them traversing the tree. Let $\mathbf{X} \in \Re^{|S| \times|M|}$ be the original feature set (dataset) and $\mathbf{F} \in \Re^{|S| \times|Q|}$ the induced feature set, where $|S|,|M|$ and $|Q|$ correspond to the number of samples, the number of original features, and the number of induced features of the dataset, respectively. Next, the clusters $q_{j} \in \mathbf{Q}$ are treated as features of the feature set $\mathbf{F}$. Each $f_{i j} \in \mathbf{F}$ equals to 1 if the sample $i \in \mathbf{S}$ is contained in the cluster (node) $q_{j}$ and 0 otherwise.

Furthermore, in order to generate a better feature representation the cluster nodes of every tree are filtered based on the volume of the samples they contain. The nodes close to the root are not so informative as they contain nearly all the samples of a dataset. By discarding the nodes containing more than $p \%$ of the samples, the number of generated features is reduced making the method more efficient. Based on the value of $p$ one could keep almost all the nodes except for the nodes associated with the roots or discard a large amount of nodes keeping this way almost only the leaves. The proposed approach is denoted as ERCP (Extremely Randomized Clustering tree-Paths). In Fig. 2, the feature induction approach is shown.

The proposed feature representation is rationally more informative than the original one. The induced features are generated by computing clusterings over the whole dataset and therefore information from the whole instance space is exploited. Samples that are outliers in the dataset can be discriminated easily, as splitting an outlier from the rest of the dataset rationally leads to large variance reduction. In addition, regions of the instance space with high variance will lead to longer paths in the trees, thereby making the procedure adaptive towards the difficulty of the instances considered. Moreover, one can control the growing of the trees by setting specific stopping criteria.

It has to be noted that a similar encoding could be produced by any hierarchical clustering method. In addition, the general idea of generating features by partitioning the training space can be connected to matrix partitioning approaches and methods based on hashing. For example, in Locality Sensitive Hashing several hyperplanes randomly partition the training space, generating in this way partition-based features. However, the employment of ERT is beneficial. First, ERT is a tree ensemble method, and therefore it is robust to small perturbations in the data. Moreover, another advantage is that the tree ensembles can generally treat both numerical and non-numerical values, making the method more easily applied and robust. In addition to that, in contrast to many other methods, it offers a natural way to deal with missing values by distributing instances with a missing split value over all branches or by selecting at random one branch to follow. The method is also performed in an inductive manner. After the training, the model can handle any new data without any need of the training set. Furthermore, it is expected that a greater number of samples will lead to bigger trees in the forest. The pro- 
posed representation will be therefore larger but also very sparse. This way, the application of our approach to modern online systems as well as systems that handle large scale data is feasible. Moreover, the proposed approach can be used in a supervised way, exploiting information from existing labels.

\subsection{Random sampling of the target space}

The method presented in Section 3.2 (ERCP) is further improved focusing on the reduction of the computational cost without affecting the performance. More specifically, the idea of random sampling the space where the variance reduction is computed during the learning of the ensembles is applied. Hereafter, the space where the variance reduction is computed is denoted as target space. The idea of applying random sampling to the target space has been investigated for supervised learning tasks but not for unsupervised tasks or feature induction. In tree-ensemble learning there is always an input and a target space. The input space $\mathbf{X} \in \Re^{|S| \times|M|}$ consists of $|S|$ instances described by $|M|$ features and the target space consists of the same features in unsupervised learning or the labels in supervised learning. The proposed feature induction method $E R C P$ is based on multi-output ensemble learning. More precisely, the trees are constructed by computing the sum of variances at each node and for each candidate split. When it comes to high dimensional representations (i.e., datasets with many features) the computation of the sum of variances over the whole set could be a serious burden for the efficiency of the method.

Here, we address this computational bottleneck by extending the feature selection strategy used in the input space to the target one. In particular, for unsupervised learning, for every tree $\kappa$ of the forest a random subset (w.r.t. the columns) $\mathbf{X}_{\kappa} \in \Re^{|S| \times\left|M_{\kappa}\right|}$ is sampled. Next, each tree $\kappa$ is grown over the learning set $\left(\mathbf{X}, \mathbf{X}_{\kappa}\right)$ instead of the $(\mathbf{X}, \mathbf{X})$. More precisely, to determine a split, all columns in $\mathbf{X}$ are considered while the variance reduction is computed over the $\mathbf{X}_{\kappa}$. In Algorithm 1, the feature induction strategy with random sampling is described in detail. For a single tree, the computational complexity of the original ERCP method is $O\left(T M_{s} N \log N\right)$, where $N$ is the number of samples, $M_{s}$ is the number of features selected as splitting candidates, and $T$ is the number of the targets. In the unsupervised setting, the input space is the same as the target one and thereby $T=M$. Following the random sampling strategy, the computational complexity is turned into $O\left(M_{\kappa} M_{s} N \log N\right)$, where $M_{\kappa}$ is the number of the sampled targets. Clearly, the proposed scheme is computationally more efficient as $M_{\kappa}<M$.

\subsection{Mining Interaction Data}

As mentioned before, the relations between two entities that interact with each other are represented as a network (here, equally referred to as a graph). Let 


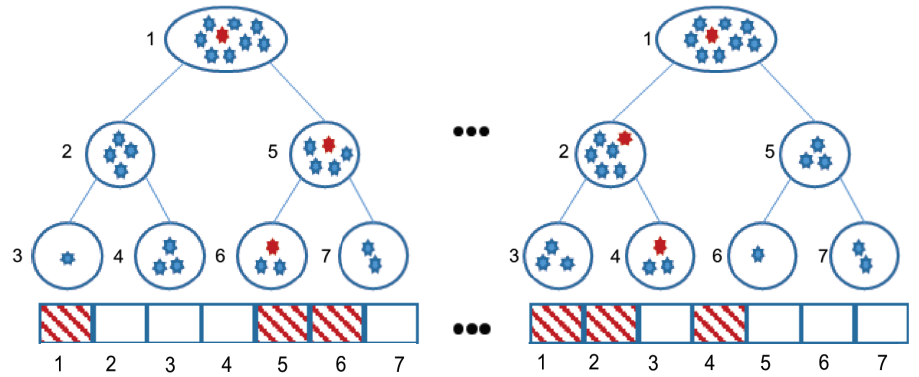

Fig. 2 Illustration of the proposed approach. The example associated with the induced feature vector is depicted as red.

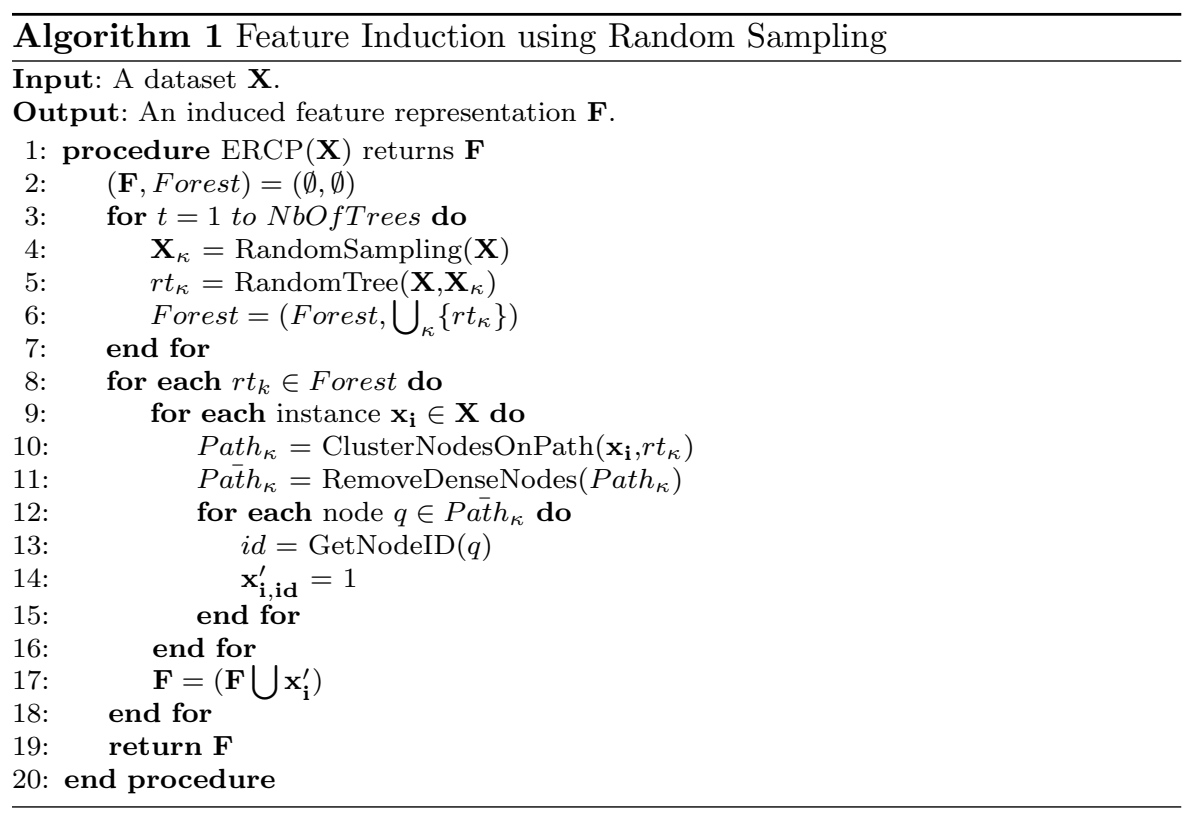

$G$ define a network with two finite sets of nodes $N_{r}=\left\{n_{r 1}, \cdots, n_{r\left|N_{r}\right|}\right\}$ and $N_{c}=\left\{n_{c 1}, \cdots, n_{c\left|N_{c}\right|}\right\}$. Each node of the network is described by a feature representation. The network corresponds to a bipartite graph over the two sets of nodes $N_{r}$ and $N_{c}$. The interactions between $N_{r}$ and $N_{c}$ are modeled as edges connecting the nodes and are represented in the adjacency matrix $\mathbf{Y} \in \Re^{\left|N_{r}\right| \times\left|N_{c}\right|}$. Every item $y(i, j) \in \mathbf{Y}$ is equal to 1 if an interaction between items $n_{r i}$ and $n_{c j}$ exists and 0 otherwise. Homogeneous graphs defined on only one type of nodes can be obtained as a particular case of the aforementioned general framework by considering two identical sets of nodes (i.e., $N_{r}=N_{c}$ ).

There are mainly two methodologies to apply a learning technique in the aforementioned framework, the local approach [33] and the global one [34]. A discussion of the two aforementioned approaches takes place in [35]. Following the local approach one should first decompose the data into separate 
(traditional) feature vector representations. Let $\mathbf{X}_{\mathbf{r}} \in \Re^{\left|N_{r}\right| \times\left|M_{r}\right|}$ be the representation of the $N_{r}$ set of nodes and $\mathbf{X}_{\mathbf{c}} \in \Re^{\left|N_{c}\right| \times\left|M_{c}\right|}$ be the representation of the $N_{c}$ set of nodes. Next, each representation's learning task is solved independently, and the results are then combined. In the global approach, one model is built, incorporating the two interaction sets in a unified framework. The applied learning technique is adapted so that it can handle the structured representation directly. The most straightforward approach in this case is to build the Cartesian product of the feature vectors that correspond to the nodes of the network. Next, a common learning method can be applied directly. For example, one could address the problem of interaction prediction in a drug protein network as a classification task. Following the local approach, one multi-output classifier is built over the set of drugs $\left(N_{r}\right)$ and another multi-output classifier is built over the set of proteins $\left(N_{c}\right)$. The results of the two classifiers are then combined yielding the final predictions. Following the global approach, a single-output classifier is built over the Cartesian product of the two sets of nodes (i.e., feature vectors that correspond to drugs and proteins). In Fig. 3 a representation of the two settings is illustrated.

The proposed approach is a combination of the local and the global approach. The bipartite graph is first decomposed into two separate sets of nodes. For example in a drug-protein interaction network one has a set of nodes corresponding to drugs and one corresponding to proteins. Each set of nodes $N_{r}$ or $N_{c}$ is represented by a feature set $\mathbf{X}_{\mathbf{r}} \in \Re^{\left|N_{r}\right| \times\left|M_{r}\right|}$ or $\mathbf{X}_{\mathbf{c}} \in \Re^{\left|N_{c}\right| \times\left|M_{c}\right|}$, respectively. Next, two feature sets $\mathbf{F}_{\mathbf{r}} \in \Re^{\left|N_{r}\right| \times\left|Q_{r}\right|}$ and $\mathbf{F}_{\mathbf{c}} \in \Re^{\left|N_{c}\right| \times\left|Q_{c}\right|}$ are induced by applying ERCP on $\mathbf{X}_{\mathbf{r}}$ and $\mathbf{X}_{\mathbf{c}}$ respectively, as described in Sec. 3.2. The new high dimensional feature representation of the nodes is then transferred to a lower dimensional space $d\left(d_{r} \ll\left|Q_{r}\right|, d_{c} \ll\left|Q_{c}\right|\right)$. This transformation is performed by embedding the data into a linear or non-linear subspace of lower dimensionality (PCA, Kernel-PCA,...). Next, a global data representation is built as the Cartesian product of the two feature spaces, as demonstrated in Fig. 3(b). More precisely, a feature vector is generated for each pair of nodes as the concatenation of the feature vectors corresponding to the nodes of the pair. To this end, a global representation $\mathbf{F}^{\prime}$ is yielded, where $\mathbf{F}^{\prime} \in \Re^{\| \mid}\left|N_{r}\right| *\left|N_{c}\right||| \times\left\|\left|d_{r}\right|+\left|d_{c}\right|\right\|$. Hereafter, the proposed model for mining interaction networks is denoted as MINET. In Fig. 4, the proposed model $(M I N E T)$ is displayed.

\subsection{Supervised network mining}

In supervised network mining usually the input is composed by pairs of instances and the values of interest are the possible interactions between the instances. The task of predicting these values is referred to as interaction prediction. For example, in a drug protein interaction network, one aims to predict the possible interactions between a drug and the proteins that are present in the network. In supervised learning, interaction prediction can be addressed as a classification problem on pairs of instances. In that case, one has 


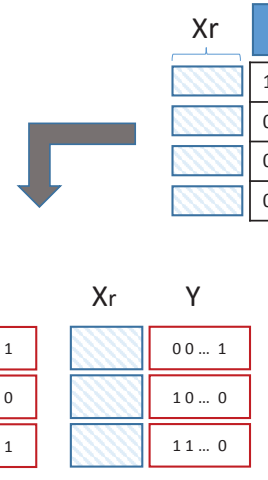

(a) Local Multi-output $-x c$
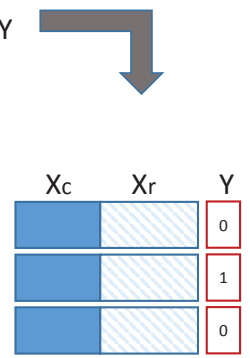

(b) Global single output

Fig. 3 A description of the two learning approaches.

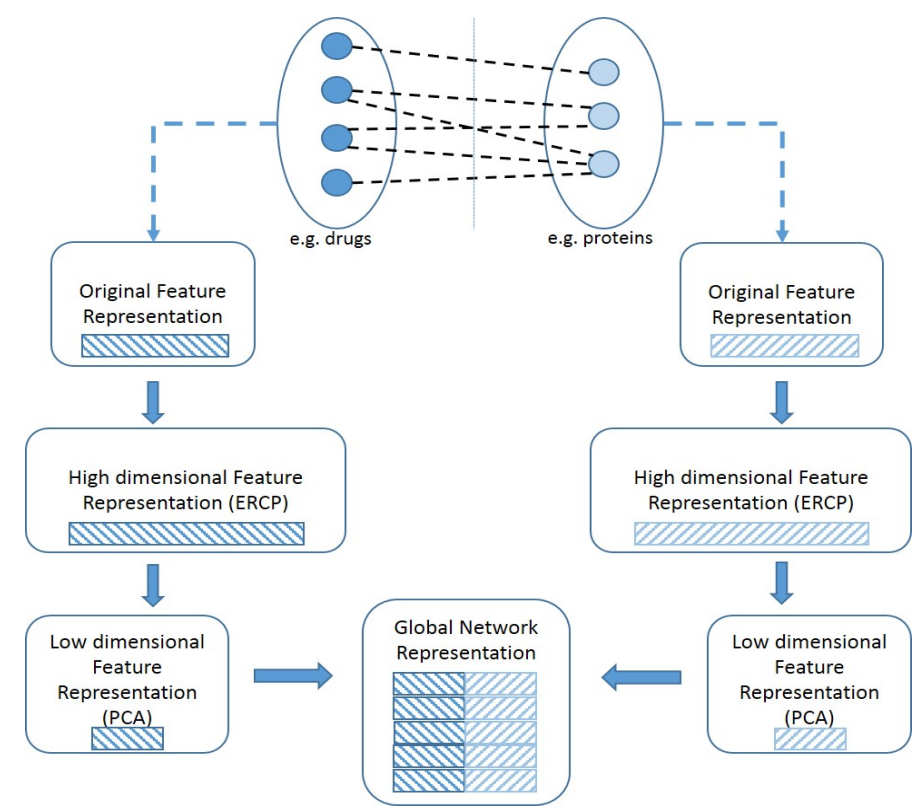

Fig. 4 A description of the proposed model (MINET).

to take into consideration two main types $[36,37]$ of predictions. Predictions where the test is a pair of totally unseen instances (e.g., drugs, proteins, genes, etc.) and predictions where one of two instances of the pair is included in the learning procedure. Normally, predictions on pairs of totally unseen instances are significantly more difficult. In particular, the supervised mining setting of our framework is displayed in Fig. 5. The $\left(L_{r} \times L_{c}\right)$ corresponds to the interaction matrix of the network that is available during the training process. It consists of the interactions (i.e., $\mathbf{Y}$ ) that are used in the learning procedure. The learner (e.g., classifier, regressor) is built on the instances included in the 


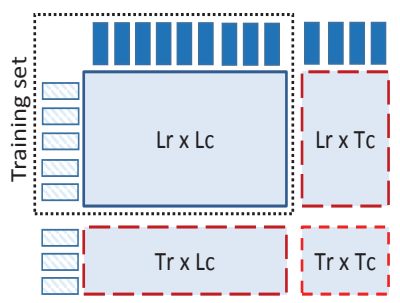

Fig. 5 The supervised mining setting of an interaction network.

$\left(L_{r} \times L_{c}\right)$ setting. The training set consists of the feature vectors and the targets that are associated with the instances included in the $\left(L_{r} \times L_{c}\right)$ setting. As one considers supervised learning, the mining setting can be divided into 3 sub-settings.

- Learned rows - Test columns $\left(L_{r} \times T_{c}\right)$ : predictions involving row instances (e.g., drugs) that have been included in the learning procedure and unseen (test) column instances (e.g., proteins).

- Test rows - Learned columns $\left(T_{r} \times L_{c}\right)$ : predictions involving unseen (test) row instances (e.g., drugs) and column instances (e.g., proteins) that have been included in the learning procedure.

- Test rows - Test columns $\left(T_{r} \times T_{c}\right)$ : predictions involving unseen (test) row instances (e.g., drugs) and unseen (test) column instances (e.g., proteins).

Following the inductive setup, here we do not consider the semi-supervised problem of performing predictions between pairs of samples that are both included in the learning procedure $\left(L_{r} \times L_{c}\right)$.

\section{Experimental Evaluation}

\subsection{Datasets}

The potential of the proposed feature induction method (ERCP) was affirmed also in [4], where the method was tested on 16 UCI datasets [38]. Here, we use datasets that correspond to interaction networks, as we focus on the network setting. In particular, 6 heterogeneous interaction datasets [3] were employed from the field of biomedicine.

In particular:

- E. coli regulatory network (ERN) [39]. This heterogeneous network consists of 179256 pairs of 154 transcription factors (TF) and 1164 genes of E. coli $(154 \times 1164=179256)$. The feature vectors that represent the two sets consist of 445 expression values.

- S. cerevisiae regulatory network (SRN) [40]. This heterogeneous network is composed by interactions between TFs and their target S. cerevisiae genes. It is composed of 205773 pairs of 1821 genes and 113 TFs. The input 
Table 1 The datasets used in the evaluation procedure are presented. The table contains the number of rows, columns, and their corresponding features. Information about the number and percentage of existing interactions in each network is also disclosed.

\begin{tabular}{|l|c|c|c|}
\hline Dataset & rows $\times$ columns & Nb of Features & Nb of interactions \\
\hline ERN & $1164 \times 154$ & $445-445$ & $3293 / 179256(1.8 \%)$ \\
SRN & $1821 \times 113$ & $9884-1685$ & $3663 / 205773(1.7 \%)$ \\
DPI-E & $664 \times 445$ & $664-445$ & $2926 / 295480(1 \%)$ \\
DPI-I & $204 \times 210$ & $204-210$ & $1476 / 42840(3.4 \%)$ \\
DPI-G & $95 \times 223$ & $95-223$ & $635 / 21185(3 \%)$ \\
DPI-N & $26 \times 54$ & $26-54$ & $90 / 1404(6.4 \%)$ \\
\hline
\end{tabular}

features are 1685 expression values. For genes, motifs features were concatenated to the expression values yielding feature vectors of 9884 values.

- Drug-protein interaction networks (DPIs) [41]. These datasets are 4 drug-protein interaction networks in which proteins belong to four pharmaceutically useful classes: enzymes (DPI-E), ion channels (DPI-I), Gprotein-coupled receptors (DPI-G) and nuclear receptors (DPI-N). The input feature vectors for proteins represent the similarity with all proteins in terms of sequence and the input feature vectors for drugs the similarity with all drugs in terms of chemical structure.

The summarization of the datasets and their characteristics is shown in Table 1.

\subsection{Experimental Setup}

The experimental evaluation starts by testing the proposed network mining method MINET, presented in Section 3.4, in an unsupervised setting. The proposed extensions over the unsupervised feature induction method $(E R C P)$ are then evaluated. The two proposed schemes (i.e., filtering scheme and random sampling of the target space), presented in Sections 3.2 and 3.3, are evaluated separately in order to properly measure the performance of each one of them.

The global representation was constructed as described in Sec. 3.4. It consists of all the possible pairs of network nodes. The known interactions or non-interactions between the nodes of the network were coded as 1 and 0 , respectively. In the unsupervised setting, they were used as ground truth without taking part in the learning process. Then, the performance of a k-NN algorithm applied to that global representation is measured. More precisely, what is measured is how well the data are distributed in the space and how "pure" the neighborhoods formed by k-NN are. The underlying idea is that instances with the same class should get a similar feature representation, even though that class information is not used in the construction of the features.

Next, the MINET is evaluated in a supervised setting. In the supervised setting, the linear SVM classifier is employed to evaluate the proposed network 
mining method. The supervised learning procedure was evaluated in all 3 prediction settings (i.e., $T_{r} \times L_{c}, L_{r} \times T_{c}$, and $T_{r} \times T_{c}$ ) as described in Section 3.5. In $T_{r} \times L_{c}$ and $L_{r} \times T_{c}$ a 5 -fold cross validation (CV) setting on nodes (i.e., $\mathrm{CV}$ on rows and $\mathrm{CV}$ on columns of the interaction matrix, respectively) was applied. In $T_{r} \times T_{c}$, a 5 -fold CV setting on both rows and columns was applied, excluding one row fold and one column fold from the learning set, and using their combined interactions as test set. The global representation based on the original features is denoted as Global Network Representation $(G N R)$.

The evaluation measures that were employed are the area under precision recall curve (AUPR) and the area under the receiver operating characteristic curve (AUROC). A PR curve is defined as the Precision $\left(\frac{T P}{T P+F P}\right)$ against the Recall $\left(\frac{T P}{T P+F N}\right)$ at various thresholds. A ROC curve is defined as the true positive rate $\left(\frac{T P}{T P+F N}\right)$ against the false positive rate $\left(\frac{F P}{F P+T N}\right)$ at various thresholds. The true-positive rate is the same as recall, and is also known as sensitivity, the false-positive rate is also known as (1 - specificity). Since the data are heavily class imbalanced, other measures such as common accuracy were avoided.

The number of trees used in the ensembles for all the compared methods was set equal to 200. This number was selected as a trade-off between accuracy and computational efficiency. All trees were unpruned, and the minimal number of instances a leaf has to cover was set equal to 5 . Typical values used in ensemble learning, such as 1 or 2, would be unusual in our setting, as we consider the nodes of the trees as clusters. The method is not sensitive to those values. The variance over the target set was computed as the sum of the variances over the individual targets.

\subsection{Results and Discussion}

In Fig. 6, a visualization of all the datasets used in the evaluation procedure is displayed by projecting the data in a 2-dimensional (2D) space using PCA. As it was described, every interaction network consists of 2 interaction sets. For example, in a drug-protein interaction network there is a dataset that corresponds to drugs and a dataset that corresponds to proteins. In order to better demonstrate the visualization property of the basic proposed feature induction method $(E R C P)$, we applied the $E R C P$ in both interaction sets of every network and then projected them in $2 \mathrm{D}$. The yielded projections were compared to corresponding $2 \mathrm{D}$ projections of the original data. As it is reflected in Fig. 6, the generated data distributions after applying PCA to the original data generally do not follow any manifold. Moreover, in most cases they are similar to a common random projection (e.g., DPI-E:drugs, DPI-I:drugs, DPI-G:drugs, DPI-N:drugs, ERN:genes, ERN:TFs, SRN:genes, SRN:TFs). ERCP leads to manifold detection and better data distributions. It manages to compress the data to only 2 dimensions removing noise and revealing hidden structures. The only exceptions are the SRN:genes and the 
DPI-N:proteins where both representations (i.e., original data, $E R C P$ ) are similar to random projections.

For further analysis, two datasets were picked, the genes from the ERN network and the proteins from the DPI-E network. PCA, Kernel-PCA [42], Laplacian Eigenmaps [43], and T-SNE [44] [14] were applied to both original feature representations of these datasets and the ones generated by ERCP. Kernel-PCA is a kernel extension of PCA, achieving non-linear transformation of the data. Laplacian Eigenmaps constructs a nearest neighbors graph from the data. Next, the transformation of the data is achieved using spectral decomposition of the corresponding graph Laplacian. T-SNE computes joint probabilities between data points based on their similarities and minimizes the Kullback-Leibler divergence between the joint probabilities of the low-dimensional embedding and the high-dimensional data.

The results are demonstrated in Figs. 7 and 8. As it is shown, ERCP generates better data representations in most cases. In particular, as it is shown in Fig. 8, apart from the Laplacian Eigenmaps, all the other methods generate projections similar to a random projection when applied to the original data. When it comes to Fig. 7, applying PCA to the original data also succeeds in revealing a hidden structure in the data. It is also interesting to notice that in both figures the projection achieved by applying Laplacian Eigenmaps on the original data is similar to the projections achieved by ERCP in combination to PCA or Kernel-PCA. This is based on the fact that the proposed approach similar to Laplacian Eigenmaps takes into account the intrinsic geometry of the data. Different from Laplacian Eigenmaps, $E R C P$ is based on tree ensembles and not a nearest neighbors graph, inheriting thereby all the advantages of tree ensemble learning (i.e., inductive setup, scalability, etc.).

The k-NN algorithm was employed in a 10-fold CV to evaluate the unsupervised MINET. The MINET was compared to GNR and GNR-KPCA. In $G N R-K P C A$, Kernel-PCA was applied to the original features of each nodeset and then the global representation (Cartesian product) was formed. GNR with PCA (GNR-PCA) was also used, showing however no difference than $G N R$ and therefore omitted. The AUROC results of all 6 network datasets are demonstrated in Fig 9 for several numbers of nearest neighbors. The optimal number of nearest neighbors in $\mathrm{k}-\mathrm{NN}$ varies depending on the dataset. Thus, we follow the performance of the compared methods across several numbers of nearest neighbors. It is shown that as we set high numbers of nearest neighbors, the results drop for the smallest dataset (i.e., dpin) but increase for the largest datasets (i.e., dpie, ern, srn). This is logical as $\mathrm{k}$ in $\mathrm{k}-\mathrm{NN}$ depends on the total number of samples in a dataset. As it is reflected in Fig. 9, the MINET generally outperforms both the GNR and the KPCA-GNR, for both small and high numbers of nearest neighbors. More specifically, the MINET is better in all datasets except for DPI-E. As noted in [29,27], it is difficult to develop a method that performs best in every network. In order to properly evaluate the representations all the components were kept. In Kernel$P C A$, the $R B F$ kernel was used and the $\gamma$ parameter was tuned internally $(\gamma=\{0.0001,0.001,0.01,0.1,1\})$. To this end, it could be deducted that the 

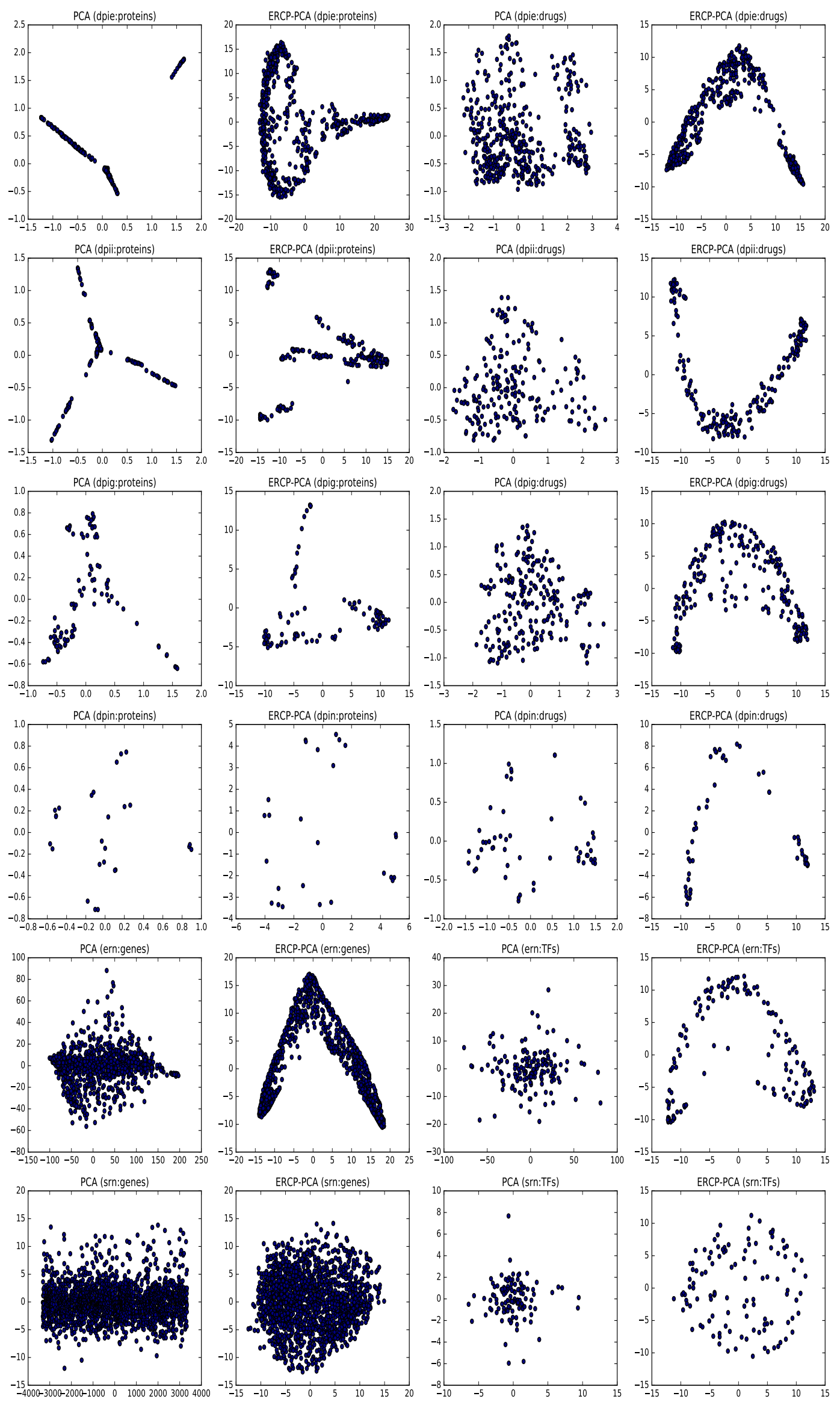

Fig. 6 2D projections of both interaction sets of all the networks used in our evaluation procedure. 

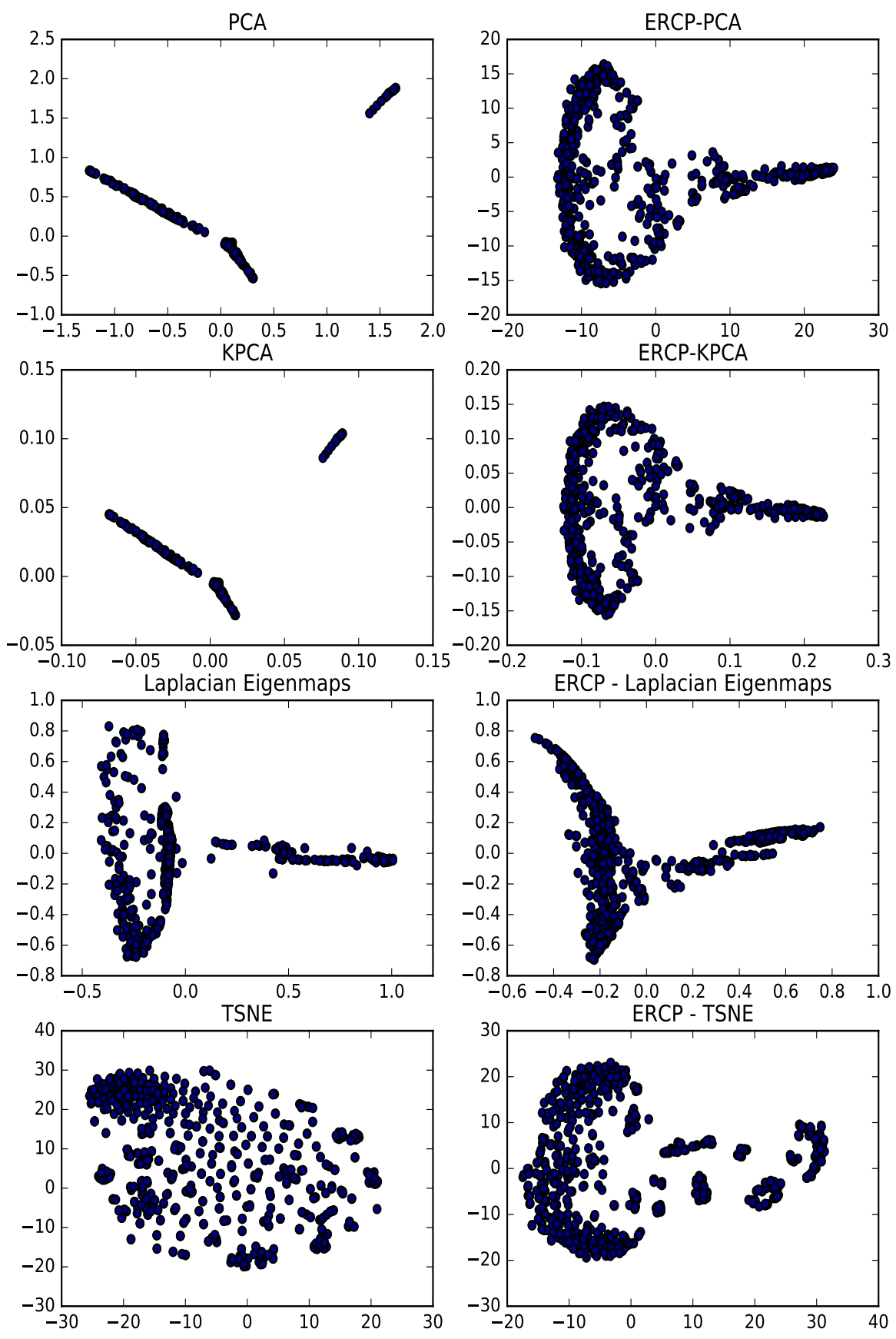

Fig. 72 D projection of the data that correspond to the proteins of the DPI-E dataset. 

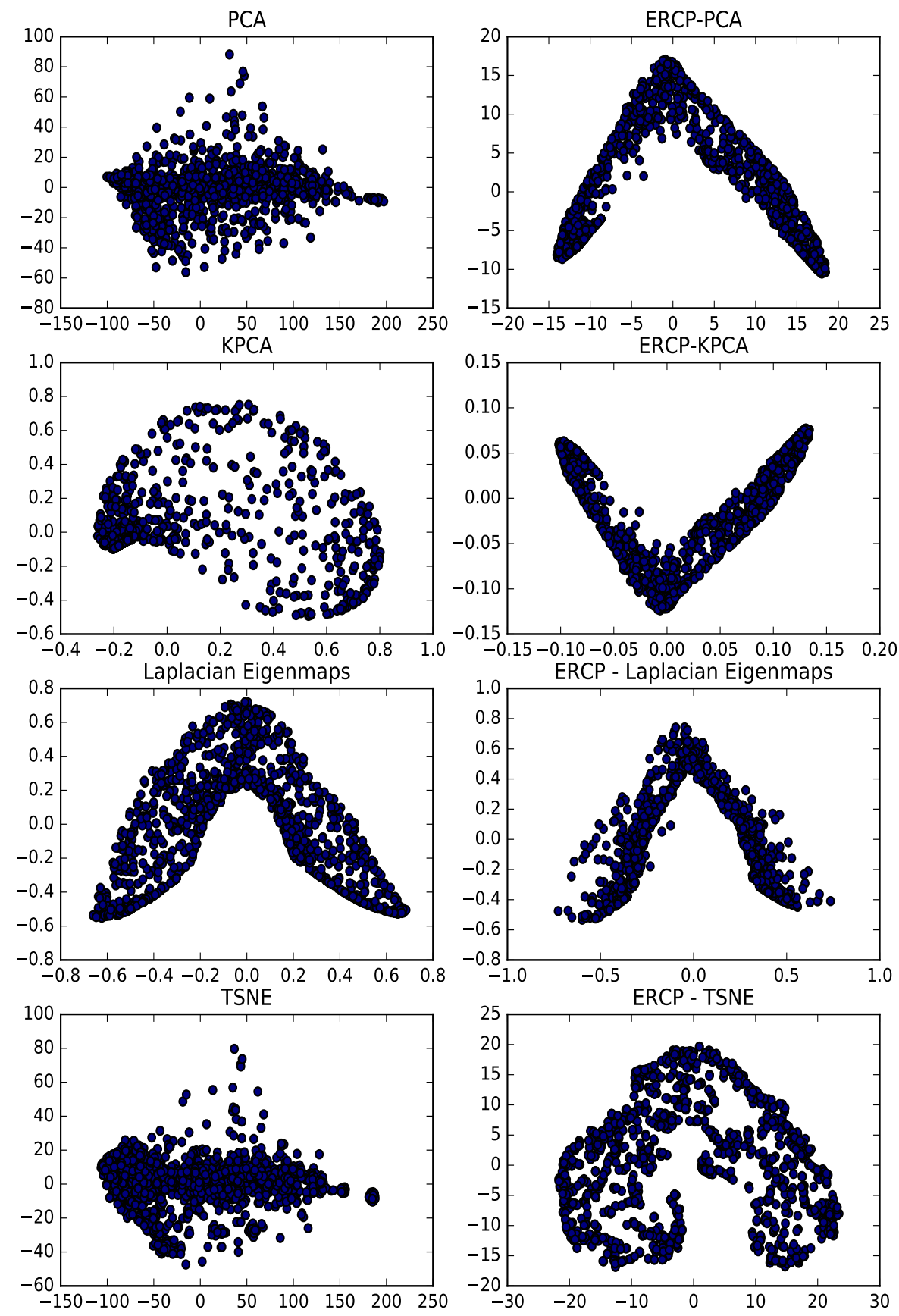

Fig. 8 2D projection of the data that correspond to the genes of the ERN dataset. 

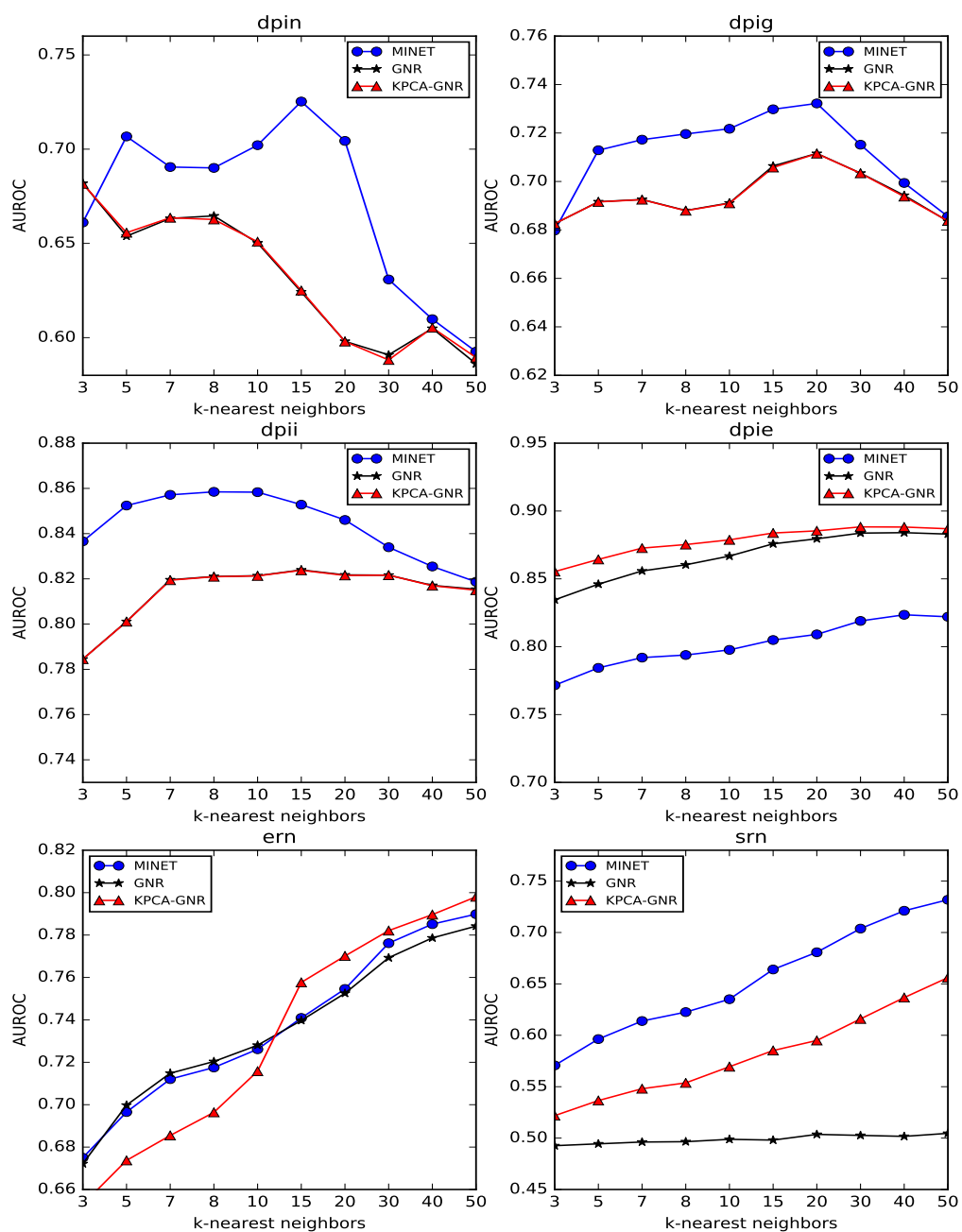

Fig. 9 AUROC results for the compared approaches.

representation generated by our approach is characterized by more pure neighborhoods.

Next, the effects of the node-filtering scheme, the random sampling, and the dimensionality reduction were measured. In Fig. 10, the effect of the clusternode weighing (i.e., refinement of the features) is disclosed. More precisely, 
k-NN $(k=5)$ was applied to the MINET representation and experiments were repeated, keeping each time only the induced features that correspond to the nodes containing less than $p *|S|$ samples, where $|S|$ is the total number of the samples of a dataset. As it is shown, many features can be removed without any significant effect in the performance, making this way the method computationally more efficient. A small $p$ leads to the removal of many nodes. Although this is beneficial in terms of computational efficiency it can lead to information loss. In case of datasets that lack variance, even the leaves of a tree could contain many samples. As reflected in Fig. 10, selecting $p=0.6$ is a relatively safe option. It guarantees that the root nodes and non-informative nodes close to them will be discarded, without risking the loss of valuable information.

In Fig.11, the performance of MINET in terms of AUROC was measured for different values of $\operatorname{dim}_{p}$, where $\operatorname{dim}_{p} *|M|$ is the number of components kept in the dimensionality reduction step. As it is shown, reducing the dimensions does not lead to major decrease in the performance. For the DPI-E dataset the performance was even increased, probably due to noise removal.

Furthermore, in Fig.12, the performance of MINET in terms of AUROC was measured for different values of $\operatorname{rand}_{p}$, where $\operatorname{rand}_{p} *|M|$ is the number of columns sampled in every tree of the ensemble during the learning procedure. It is shown that the random sampling approach succeeds in reducing the target space during learning without any major losses in the performance.

A serious bottleneck when it comes to global network approaches is the size of the global network representation. The transferring of the data to a low dimensional space is therefore crucial. We extend the evaluation procedure by testing MINET's efficiency particularly in a low dimensional space. For this, we included two graph embedding methods [45], the Isometric mapping (Isomap) [46] and the Locally Linear Embedding (LLE) [47]. Both methods build a k-NN graph (here, the size of the neighborhoods was set to 5) and then transform the data, preserving the distances in local neighborhoods (LLE) or preserving the geodesic distances between all nodes (Isomap). For each network dataset, the two set of nodes were initially transferred to a low dimensional space. This was set to $20 \%$ of the size of the original feature vectors. For the srn network, due to its large original feature set, a value of $5 \%$ was used. Next, the Cartesian product was built. The obtained results in terms of AUROC are presented in Fig. 13. It is shown that MINET outperforms the other approaches in most cases affirming this way its efficiency. Note that here we only test MINET's efficiency after transferring the data to a low dimensional space. We cannot conclude that one approach is generally better than the others as we do not know precisely the true amount of information lost while reducing the dimensionality. It should also be clarified that approaches such as LLE, or Isomap, could be applied to our setting as well, replacing PCA.

Moreover, linear SVM was employed to evaluate the supervised version of MINET. The SVM takes as input a parameter $\mathcal{C}$ that indicates the trade-off between training error and margin. The optimal value for $\mathcal{C}$ was tuned internally from the set $\{0.01,0.1,1,10,100\}$. The evaluation was performed in a 5 -fold 

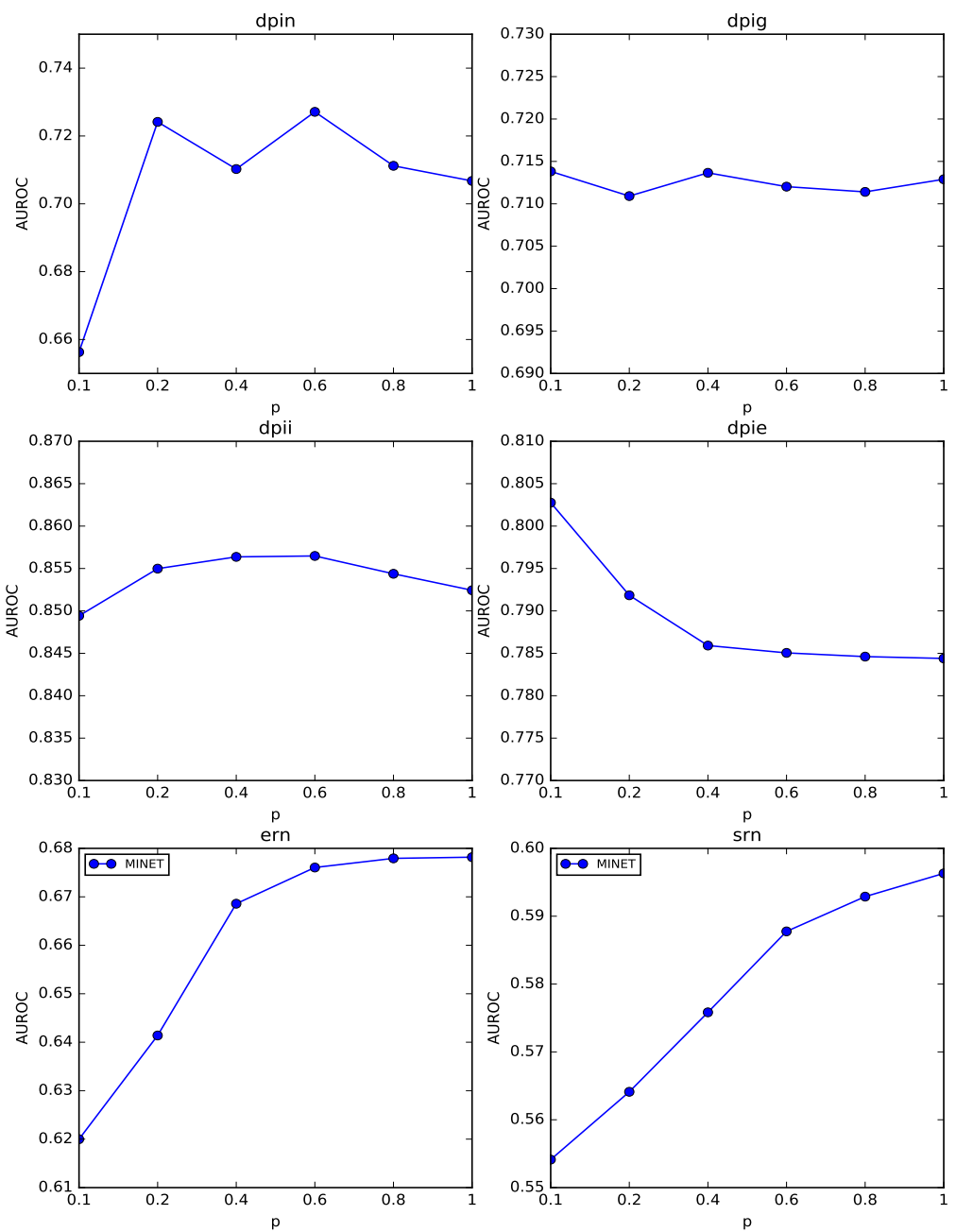

Fig. 10 AUROC results for different values of $p$. 

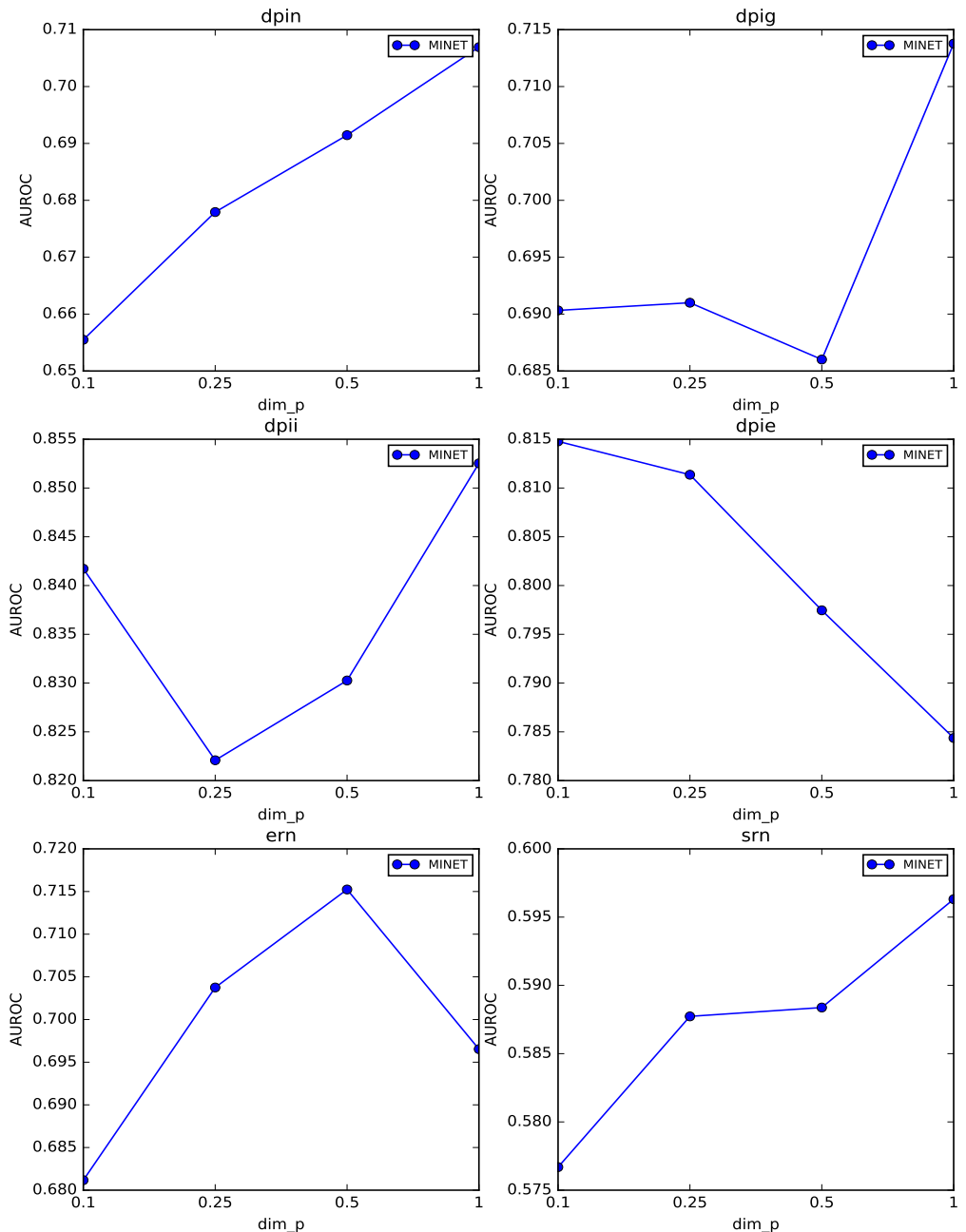

Fig. 11 AUROC results for different values of $\operatorname{dim}_{p}$. 

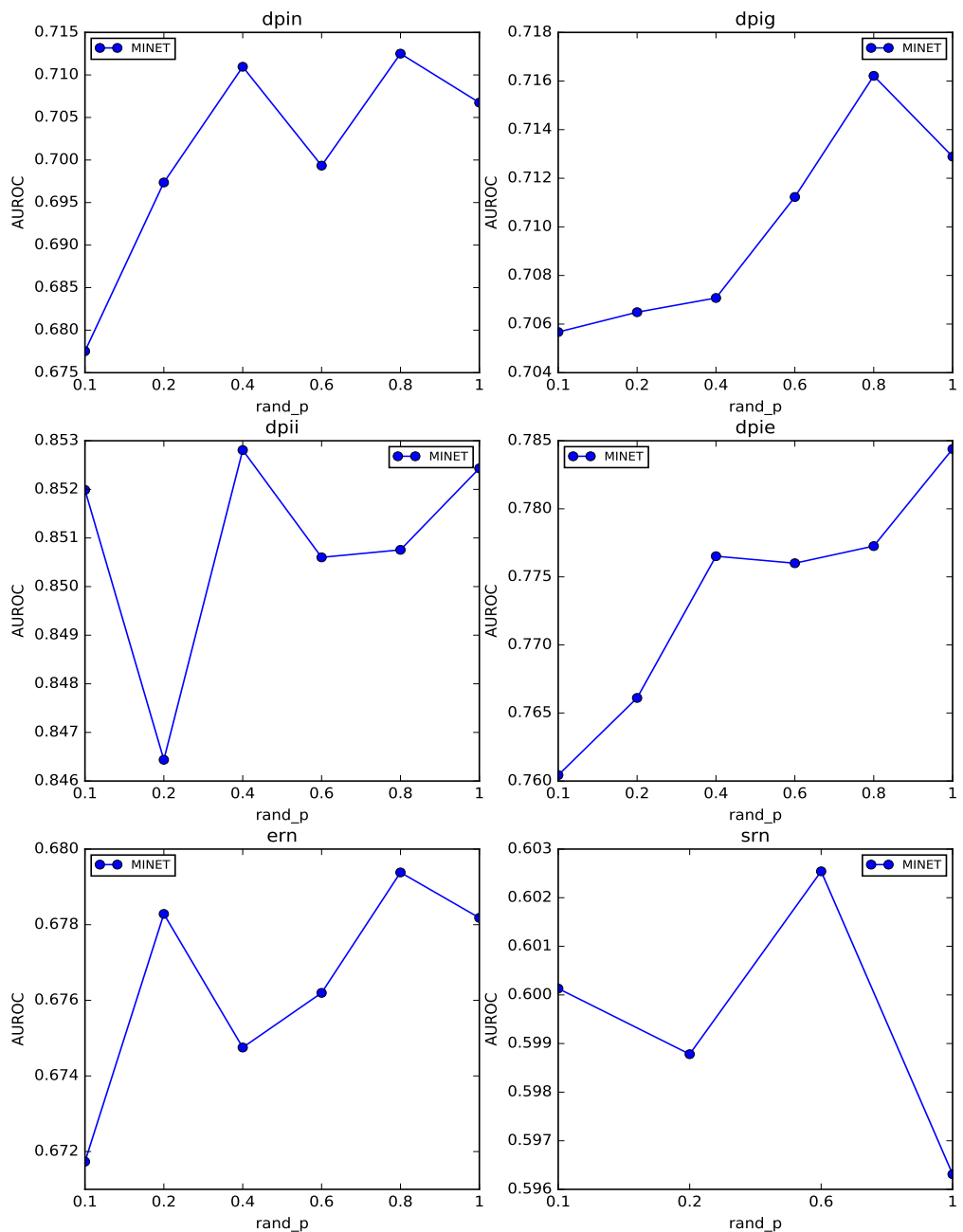

Fig. 12 AUROC results for different values of $\operatorname{rand}_{p}$. 

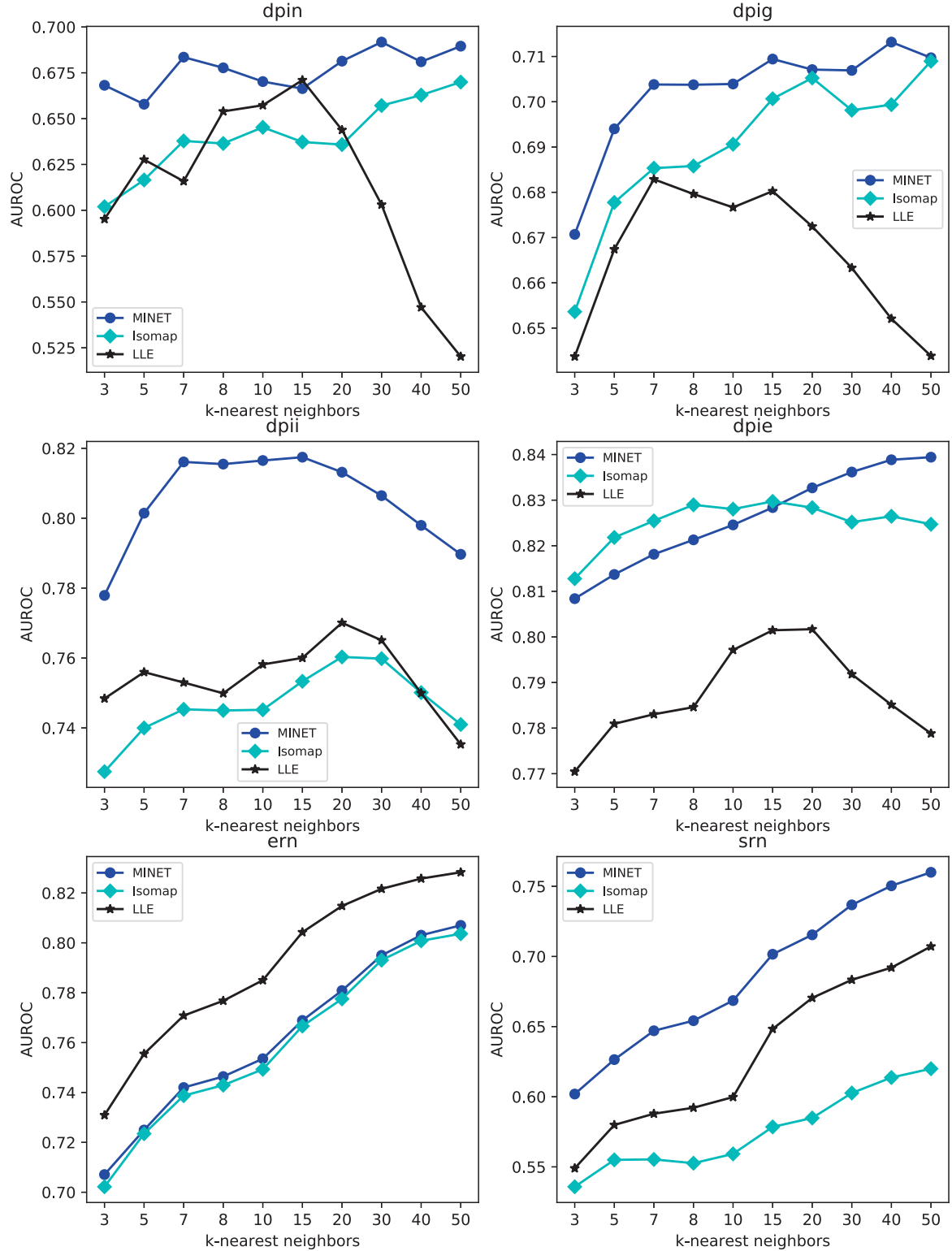

Fig. 13 AUROC results for the compared approaches. 
Table 2 AUROC measures for the compared approaches.

\begin{tabular}{|c||c|c||c|c||c|c|}
\hline \multicolumn{1}{|c||}{} & \multicolumn{2}{c||}{$T_{r} \times L_{c}$} & \multicolumn{2}{c||}{$L_{r} \times T_{c}$} & \multicolumn{2}{c|}{$T_{r} \times T_{c}$} \\
\hline Data & $G N R$ & $M I N E T$ & $G N R$ & $M I N E T$ & $G N R$ & $M I N E T$ \\
\hline DPI-N & 0.531 & $\mathbf{0 . 5 6 5}$ & 0.662 & $\mathbf{0 . 7 3 9}$ & 0.562 & $\mathbf{0 . 5 7 6}$ \\
DPI-G & 0.448 & $\mathbf{0 . 5 6 1}$ & 0.532 & $\mathbf{0 . 6 5 4}$ & 0.495 & $\mathbf{0 . 5 6 7}$ \\
DPI-I & 0.572 & $\mathbf{0 . 6 2 9}$ & 0.494 & $\mathbf{0 . 5 2 3}$ & $\mathbf{0 . 4 9 2}$ & 0.455 \\
DPI-E & 0.548 & $\mathbf{0 . 6 4 8}$ & 0.531 & $\mathbf{0 . 6 5 6}$ & 0.513 & $\mathbf{0 . 6 0 1}$ \\
ERN & $\mathbf{0 . 7 3 4}$ & 0.724 & 0.488 & $\mathbf{0 . 5 0 1}$ & 0.524 & $\mathbf{0 . 6 0 2}$ \\
SRN & $\mathbf{0 . 5 6 2}$ & 0.44 & $\mathbf{0 . 4 9 1}$ & 0.462 & 0.506 & 0.506 \\
\hline Average & 0.566 & $\mathbf{0 . 5 9 5}$ & 0.533 & $\mathbf{0 . 5 8 9}$ & 0.515 & $\mathbf{0 . 5 5 1}$ \\
Average Ranks & 1.67 & $\mathbf{1 . 3 3}$ & 1.83 & $\mathbf{1 . 1 7}$ & 1.75 & $\mathbf{1 . 2 5}$ \\
\hline
\end{tabular}

CV instead of 10 for computational reasons. The AUROC and AUPR results are demonstrated in Tables 2 and 3, respectively. It is shown that supervised MINET outperforms the GNR in terms of both AUROC and AUPR. MINET outperforms $G N R$ in terms of both average results and average ranks for all three settings $\left(T_{r} \times L_{c}, L_{r} \times T_{c}\right.$, and $\left.T_{r} \times T_{c}\right)$. A Wilcoxon Signed-Ranks Test finds the AUROC results significant with a p-value $=0.02(p=0.02<0.05)$. In particular, in DPI-G and DPI-E, MINET surpass its competitor by more than $10 \%$ in $T_{r} \times L_{c}$ and $L_{r} \times T_{c}$ settings and more than $7 \%$ in $T_{r} \times T_{c}$ setting. A Wilcoxon Signed-Ranks Test finds the AUPR results not significant. The AUPR results are generally very low as the interaction prediction task is very challenging. The high sparsity and the existing class imbalance in the label sets make the task of linear separation of the data very difficult.

The problem of class imbalance is present in mining interaction networks as generally the number of the non-interactive nodes is much higher than the number of the interactive ones [37]. Class imbalance often harasses the predictive performance of applied classifiers [48]. The employment of a powerful non-linear classifier in combination with a technique to handle class imbalance could improve the results. However, reaching the optimal performance in interaction prediction by employing a state of the art classifier or by tuning all the parameters of our model in a grid search was not the goal of this work.

In general, it can be concluded that the potential of MINET was affirmed. Both supervised and unsupervised versions of the proposed network mining model MINET outperformed the compared approaches.

\section{Conclusions and Future work}

Here, we proposed an efficient network representation model based on clustering tree ensembles feature induction. In particular, we improved our previous work, proposing a computationally more efficient feature induction process. The network model was also extended to supervised learning. The proposed model is computationally efficient, inductive, interpretable and it can handle various types of data and missing values as it inherits all the advantages of the 
Table 3 AUPR measures for the compared approaches.

\begin{tabular}{|c||c|c||c|c||c|c|}
\hline \multicolumn{1}{|c||}{} & \multicolumn{2}{c||}{$T_{r} \times L_{c}$} & \multicolumn{2}{c||}{$L_{r} \times T_{c}$} & \multicolumn{2}{c|}{$T_{r} \times T_{c}$} \\
\hline Data & $G N R$ & $M I N E T$ & $G N R$ & $M I N E T$ & $G N R$ & $M I N E T$ \\
\hline DPI-N & 0.201 & $\mathbf{0 . 2 0 7}$ & 0.174 & $\mathbf{0 . 2 1 1}$ & 0.081 & $\mathbf{0 . 0 8 9}$ \\
DPI-G & 0.029 & $\mathbf{0 . 0 4 3}$ & 0.033 & $\mathbf{0 . 0 5 3}$ & 0.039 & $\mathbf{0 . 0 4 2}$ \\
DPI-I & 0.138 & $\mathbf{0 . 1 5 7}$ & 0.035 & $\mathbf{0 . 0 4}$ & $\mathbf{0 . 0 5}$ & 0.033 \\
DPI-E & $\mathbf{0 . 0 2 6}$ & 0.018 & 0.011 & $\mathbf{0 . 0 1 9}$ & $\mathbf{0 . 0 2 5}$ & 0.019 \\
ERN & 0.191 & $\mathbf{0 . 2 0 5}$ & $\mathbf{0 . 0 2 7}$ & 0.022 & 0.028 & $\mathbf{0 . 0 4}$ \\
SRN & $\mathbf{0 . 0 2 2}$ & 0.015 & $\mathbf{0 . 0 1 7}$ & 0.015 & 0.019 & 0.019 \\
\hline Average & 0.101 & $\mathbf{0 . 1 0 8}$ & 0.05 & $\mathbf{0 . 0 6}$ & 0.04 & 0.04 \\
Average Ranks & 1.67 & $\mathbf{1 . 3 3}$ & 1.67 & $\mathbf{1 . 3 3}$ & 1.58 & $\mathbf{1 . 4 2}$ \\
\hline
\end{tabular}

decision tree ensembles. We empirically evaluated the proposed method using datasets that represent heterogeneous interaction networks. The effectiveness of the proposed method was confirmed by outperforming the compared approaches.

Possible topics for future research include the application of the proposed method to interaction datasets from various fields and the application of several machine learning algorithms to the generated feature representation. Furthermore, the supervised version of the proposed method could be extended towards addressing the problem of class imbalance.

\section{References}

1. Sun, Y., Han, J.: Mining Heterogeneous Information Networks: Principles and Methodologies. Synthesis Lectures on Data Mining and Knowledge Discovery 3, 2, 1-159 (2012)

2. Sun, Y., Han, J.: Mining heterogeneous information networks: a structural analysis approach. ACM SIGKDD Explorations Newsletter 14, 2, 20-28 (2013)

3. Schrynemackers, M., Wehenkel, L., Babu, M. M., Geurts, P.: Classifying pairs with trees for supervised biological network inference. Molecular BioSystems, 11, 8, 2116-2125 (2015)

4. Pliakos, K., Vens, C.: Feature Induction and Network Mining with Clustering Tree Ensembles. New Frontiers in Mining Complex Patterns. (NFMCP 2016). Lecture Notes in Computer Science, 10312, 3-18, (2017)

5. Blockeel, H., De Raedt, L.: Top-down induction of first-order logical decision trees. Artificial intelligence, 101, 1, 285-297 (1998)

6. Blockeel, H., De Raedt, L., Ramon, J.: Top-Down Induction of Clustering Trees. in Proc. 15th Int. Conf. on Machine Learning, 55-63 (1998)

7. Geurts, P., Irrthum, A., Wehenkel, L.: Supervised learning with decision tree-based methods in computational and systems biology. Molecular Biosystems, 5, 12, 1593-1605 (2009)

8. Agichtein, E., Castillo, C., Donato, D., Gionis, A., Mishne, G.: Finding high-quality content in social media. in Proc. ACM Int. Conf. on Web Search and Data Mining, 183$194(2008)$

9. Kocev, D., Vens, C., Struyf, J., Džeroski, S.: Tree ensembles for predicting structured outputs. Pattern Recognition, 46, 3, 817-833 (2013)

10. Breiman, L.: Random forests. Machine learning, 45, 1, 5-32 (2001)

11. Geurts, P., Ernst, D., Wehenkel, L.: Extremely randomized trees. Machine learning, 63 , 1, 3-42 (2006)

12. Joly, A., Geurts, P., Wehenkel, L.: Random Forests with Random Projections of the Output Space for High Dimensional Multi-label Classification. in Machine Learning and Knowledge Discovery in Databases (ECML PKDD), Nancy, France, 607-622 (2014) 
13. Yan, S., Xu, D., Zhang, B., Zhang, H. J., Yang, Q., Lin, S.: Graph embedding and extensions: a general framework for dimensionality reduction. in IEEE trans. on Pattern Analysis and Machine Intelligence, 29, 1, 40-51 (2007)

14. Van Der Maaten, L., Postma, E., Van den Herik, J.: Dimensionality reduction: a comparative review. Journal of Machine Learning Research, 10, 66-71 (2009)

15. Cao, L. J., Chua, K. S., Chong, W. K., Lee, H. P., Gu, Q. M.: A comparison of PCA, KPCA and ICA for dimensionality reduction in support vector machine. Neurocomputing, 55, 1, 321-336, (2003).

16. Lanckriet, G. R., Cristianini, N., Bartlett, P., Ghaoui, L. E., Jordan, M. I.: Learning the kernel matrix with semidefinite programming. Journal of Machine learning research, 5, 27-72 (2004)

17. Shawe-Taylor, J., Cristianini, N.: Kernel methods for pattern analysis. Cambridge university press (2004)

18. Burges, C. J.: A tutorial on support vector machines for pattern recognition. Data Mining and Knowledge Discovery, 2, 2, 121-167 (1998)

19. Vens, C., Costa, F.: Random forest based feature induction. in Proc. IEEE 11th Int. Conf. on Data Mining (ICDM), 744-753 (2011)

20. Moosmann, F., Triggs, B., Jurie, F.: Fast discriminative visual codebooks using randomized clustering forests. in Proc. 20th Conf. on Neural Information Processing Systems (NIPS), 985-992 (2006)

21. Moosmann, F., Triggs, B., Jurie, F.: Randomized clustering forests for image classification. IEEE Trans. on Pattern Analysis and Machine Intelligence, 30, 9, 1632-1646 (2008)

22. Zhang, M., Wu, L.: LIFT: Multi-label learning with label-specific features. IEEE Trans. on Pattern Analysis and Machine Intelligence, 37, 1, 107-120 (2015)

23. Pliakos, K., Vens, C.: Mining Features for Biomedical Data using Clustering Tree Ensembles (under review)

24. Maetschke, S. R., Madhamshettiwar, P. B., Davis, M. J., Ragan, M. A.: Supervised, semi-supervised and unsupervised inference of gene regulatory networks. Briefings in Bioinformatics, 15, 2, 195-211 (2014)

25. Stojanova, D., Ceci, M., Malerba, D., Dzeroski, S.: Using PPI network autocorrelation in hierarchical multi-label classification trees for gene function prediction. BMC bioinformatics, 14, 1, 285 (2013)

26. Wang, Y. R., Huang, H.: Review on statistical methods for gene network reconstruction using expression data. Journal of theoretical biology, 362, 53-61 (2014)

27. Marbach, D., Costello, J. C., Kffner, R., Vega, N. M., Prill, R. J., Camacho, D. M., Allison K. R., The DREAM5 Consortium, Kellis M., Collins J. J., Stolovitzky G.: Wisdom of crowds for robust gene network inference. Nature methods, 9, 8, 796-804 (2012)

28. Hase, T., Ghosh, S., Yamanaka, R., Kitano, H.: Harnessing diversity towards the reconstructing of large scale gene regulatory networks. PLoS computational biology, 9, 11, e1003361 (2013)

29. Ceci, M., Pio, G., Kuzmanovski, V., Džeroski, S.: Semi-supervised multi-view learning for gene network reconstruction. PloS one, 10, 12, e0144031 (2015)

30. Pio, G., Ceci, M., Malerba, D., D'Elia, D.: ComiRNet: a web-based system for the analysis of miRNA-gene regulatory networks. BMC bioinformatics, 16, 9, S7 (2015)

31. Irrthum, A., Wehenkel, L., Geurts, P. Inferring regulatory networks from expression data using tree-based methods. PloS one, 5, 9, e12776 (2010)

32. Kocev, D., Ceci, M.: Ensembles of extremely randomized trees for multi-target regression. Discovery Science, 86-100 (2015)

33. Bleakley, K., Biau, G., Vert, J.P.: Supervised reconstruction of biological networks with local models. Bioinformatics, 23, 13, i57-i65 (2007)

34. Vert, J.P., Qiu, J., Noble, W.S.: A new pairwise kernel for biological network inference with support vector machines. BMC bioinformatics, 8, 10, 1 (2007)

35. Vert, J.P.: Reconstruction of biological networks by supervised machine learning approaches. Elements of Computational Systems Biology, pp. 165-188 (2010)

36. Park, Y., Marcotte, E.M.: Flaws in evaluation schemes for pair-input computational predictions. Nature methods, 9, 12, 1134-1136 (2012)

37. Schrynemackers, M., Kuener, R., Geurts, P.: On protocols and measures for the validation of supervised methods for the inference of biological networks. Frontiers in Genetics, 4, $262(2013)$ 
38. Asuncion, A., Newman, D.: UCI machine learning repository. [Online] Available: http://www.ics.uci.edu/ mlearn/MLRepository.html

39. Faith, J. J., Hayete, B., Thaden, J. T., Mogno, I., Wierzbowski, J., Cottarel, G., Kasif, S., Collins, J. J., Gardner, T. S.: Large-scale mapping and validation of Escherichia coli transcriptional regulation from a compendium of expression profiles. PLoS biol, 5, 1, e8 (2007)

40. MacIsaac, K. D., Wang, T., Gordon, D. B., Gifford, D. K., Stormo, G. D., Fraenkel, E.: An improved map of conserved regulatory sites for Saccharomyces cerevisiae. BMC Bioinformatics, 7, 1, 1 (2006)

41. Yamanishi, Y., Araki, M., Gutteridge, A., Honda, W., Kanehisa, M.: Prediction of drug-target interaction networks from the integration of chemical and genomic spaces. Bioinformatics 24, 13, i232-i240 (2008)

42. Schölkopf, B., Smola, A., Müller, K. R.: Kernel principal component analysis. in Int Conf. on Artificial Neural Networks, 583-588 (1997)

43. Belkin, M., Niyogi, P.: Laplacian eigenmaps for dimensionality reduction and data representation. Neural computation, 15, 6, 1373-1396 (2003)

44. Van Der Maaten, L., Hinton, G.: Visualizing data using t-SNE. Journal of Machine Learning Research, 9, 2579-2605 (2008)

45. Cai, H., Zheng, V. W., Chang, K.: A comprehensive survey of graph embedding: problems, techniques and applications. IEEE Transactions on Knowledge and Data Engineering (2018)

46. Tenenbaum, J. B., De Silva, V., Langford, J. C.: A global geometric framework for nonlinear dimensionality reduction. science, 290(5500), 2319-2323 (2000)

47. Roweis, S. T., Saul, L. K.: Nonlinear dimensionality reduction by locally linear embedding. science, 290(5500), 2323-2326 (2000)

48. He H, Garcia EA.: Learning from imbalanced data. IEEE Transactions on Knowledge Data Engineering, 21, 9, 1263-1284 (2009) 